\title{
A multi-point feed increases macromixing rate by an order of magnitude in simulated multi-impeller vessels
}

\author{
Pauli Losoi * Jukka Konttinen Ville Santala \\ Faculty of Engineering and Natural Sciences, Tampere University, Hervanta campus, \\ Korkeakoulunkatu 8, Tampere, 33720, Finland \\ * Corresponding author, pauli.losoi@tuni.fi
}

\begin{abstract}
Tall vessels equipped with multiple impellers, such as many gas-liquid contactors in bioprocessing, often suffer from poor macromixing of feeds, which leads to heterogeneities and complicates the scale-up. Here, a diffusion equation was used as a simple model of macromixing in multi-impeller tanks, and the model fitted previously published experimental tracer curves. The model was then noted to predict that the macromixing time-scale is decreased to a quarter by relocating the feed point from the top to the middle. Furthermore, dividing the vessel axially in symmetric proportions and locating a feed point in the center of each compartment was found to reduce the time-scale even further. These theoretical results were put to test by compartment model simulations of $17 \mathrm{~L}$ to $22 \mathrm{~m}^{3}$ tanks with one to four impellers. Order-of-magnitude improvements were seen in the multi-impeller setups: up to 29-fold macromixing rates compared to a single-point top feed were realized with multiple feed points in a $22 \mathrm{~m}^{3}$ bioreactor stirred with four Rushton turbines. Considerable improvements were observed also in the other scales and geometries with fewer than four impellers, which suggests that the herein proposed division of feed points may greatly facilitate the scale-up of tall multi-impeller vessels.
\end{abstract}

\section{Keywords}

macromixing model, mixing time, stirred vessel, multiple impellers, scale-up, diffusion equation 


\section{Graphical abstract}

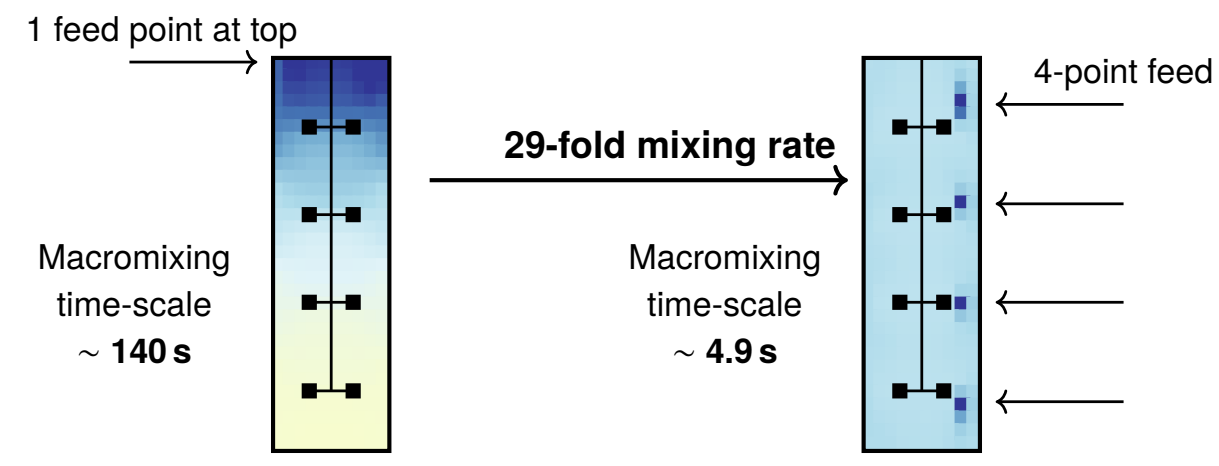

\section{Introduction}

High aspect ratio vessels with multiple impellers are used as gas-liquid contactors for their high gas hold-up and mass transfer rate. In aerobic bioprocesses they are especially prevalent and find use in the production of antibiotics, enzymes, and therapeutic proteins, to name a few. However, macromixing in a large-scale (cubic meters) multi-impeller tank tends to be severely limited, which leads to heterogeneities if the time-scale of reaction competes with that of mixing [1-5]. Some reductions in the macromixing time have been realized in experiments by relocating the single feed point from the top to the middle $[6,7]$. Additionally, both the number and placement of feed points have been found to influence mixing times in pneumatically agitated bubble column and airlift reactors [8].

To understand macromixing and its dependence on the number and location of feed points, a mathematical description is needed. The mixing of a substance fed to a stirred tank with an incompressible velocity field $v_{i}\left(\mathrm{~L} \mathrm{~T}^{-1}\right)$ is governed by transient advection and diffusion of its concentration $C\left(\mathrm{ML}^{-3}\right)$ with a diffusivity $d\left(\mathrm{~L}^{2} \mathrm{~T}^{-1}\right)$ :

$$
\underbrace{\frac{\partial C}{\partial t}}_{\text {transient }}+\underbrace{v_{i} \frac{\partial C}{\partial x_{i}}}_{\text {advection }}=\underbrace{d \frac{\partial^{2} C}{\partial x_{i}^{2}}}_{\text {diffusion }}
$$

Unfortunately, the advective part makes the equation unwieldy to analyze, and therefore experimentally validated numerical approximations, such as compartment or networksof-zones models [9-11] or computational fluid dynamics (CFD) simulations [12, 13], need to be used to model and simulate mixing. Despite their success and power in modeling and optimization of specific designs, the numerical solutions are for the most part case-specific and do not naturally lend themselves to mathematical analysis and general results.

Considering that without diffusion, no mixing would take place, it might be possible to drop the advection term of eq 1 altogether in the context of multi-impeller equipment and allow the diffusion term to model all forms of transport (advection, turbulent dispersion, 
molecular diffusion). Such a simplification would result in an analytically soluble macromixing model that might fit experimental tracer data and give insight on how the tank's geometry and the number and placement of feed points affect the macromixing time-scale. Mere diffusion as a model of mixing is similar to the classical 1D dispersioncirculation model [14-16], in which the concentration's homogenization is ultimately driven solely by the diffusive process, the dispersion. Compartment models, which are essentially discretizations of eq 1 [16], also rely on diffusion-like exchange flows, and likewise most CFD simulations model the gradient-smoothing effects of turbulence with turbulent diffusivity.

Here, the following research questions were then posed to investigate the effect of feeding on macromixing in multi-impeller vessels: First, is it possible to simplify the universal advection-diffusion equation (eq 1) to attain general results as hypothesized in the paragraph above? Second, how should a single feed point be placed to minimize the time-scale of mixing? Third, is there any notable benefit in using multiple feed points in multi-impeller configurations? Finally, how should the multiple feed points be placed?

In the context of this work, mixing refers to macromixing unless otherwise specified, and the following definitions are used to avoid confusion related to the concept of mixing time: Local mixing time refers to the time required to bring a locally measured tracer concentration within $5 \%$ of equilibrium. The time-scale of (macro)mixing is the time required to reduce the concentration's standard deviation in the whole volume to $5 \%$ of mean. The time-scale is thus a global measure of mixing efficiency [17]. For convenience, the mixing rate is defined as the inverse of the time-scale.

\section{Materials and methods}

\subsection{Dispersion model}

As the macromixing limitations of multi-impeller vessels exist predominantly along the axial dimension $[1,4,7]$, the mixing of a tracer was modeled here by reducing the transient $3 \mathrm{D}$ advection-diffusion equation (eq 1 ) to a transient $1 \mathrm{D}$ diffusion equation:

$$
\frac{\partial u}{\partial t}=d \frac{\partial^{2} u}{\partial z^{2}}
$$

where $u$ is the tracer's dimensionless concentration, $t$ time $(\mathrm{T}), d$ dispersivity $\left(\mathrm{L}^{2} \mathrm{~T}^{-1}\right)$ that covers all forms of transport, and $z$ axial coordinate (L). The axial domain was $[0, H]$, where $H$ corresponds to the height (L) of the liquid column. The domain boundaries were insulated, or in other words, they adhered to the zero-gradient or symmetry condition (no mass transfer across boundaries). The initial condition was a point-addition of tracer at $z_{0}$. By definition, the dimensionless concentration's mean is equal to 1 regardless of the injected tracer quantity. The solution to eq 2 with the described boundary and initial conditions is

$$
u(t, z)=1+2 \sum_{k=1}^{\infty} \cos \left(k \pi \frac{z_{0}}{H}\right) \cos \left(k \pi \frac{z}{H}\right) \exp \left(-k^{2} \pi^{2} \frac{d t}{H^{2}}\right)
$$


which is analogous to transient conduction of a heat impulse in an insulated domain and can be found for example in textbooks of heat transfer [18]. Previously published $[9,12,19,20]$ tracer curves were reconstructed with the proposed dispersion model (eq 3 ) by fitting the dispersion coefficient $d$ to the experimentally determined local mixing times $(95 \%$ homogeneity or equivalently $5 \%$ deviation relative to equilibrium at the measurement location). The reported tracer data points were obtained from the published figures with WebPlotDigitizer [21]. The series in eq 3 was approximated with 20 terms.

\subsection{Simulations}

The effect of the number and placement of feed points was simulated in various vessels described in the literature $[9,12,19,20]$ by extending a previously published and validated axisymmetric 2D compartment model $[7,9,10]$ to include more compartments and the tangential dimension. Additional compartments were necessary for accurate feed point placement, and the model extension followed previously published networks-of-zones models $[11,12,22]$. Briefly, the main circulation and exchange flows as defined in references [7, 9, 10], $Q_{\mathrm{C}}=N_{\mathrm{C}} n D^{3}$ and $Q_{\mathrm{E}}=N_{\mathrm{E}} n D^{3}\left(\mathrm{~L}^{3} \mathrm{~T}^{-1}\right)$, were calculated as a function of stirrer speed $n\left(\mathrm{~T}^{-1}\right)$ and impeller diameter $D(\mathrm{~L})$ with dimensionless circulation and exchange flow numbers, $N_{\mathrm{C}}$ and $N_{\mathrm{E}}$.

The impeller-wise circulation flow $Q_{\mathrm{C}}$ was divided according to the number of flow loops as in references $[11,12,22]$, and the exchange flows $Q_{\mathrm{E}}$ according to the number of compartments perpendicular to the flow $[7,9,10]$. The turbulent exchange flows were furthermore multiplied by the number of compartments parallel to the flow to complete the finite-volume discretization of the diffusive exchanges. The impeller-wise tangential circulation flow was assumed to be equal to the impeller's main circulation flow $Q_{\mathrm{C}}$ $[12,22]$, but it was divided according to the number of flow loops in the tangential direction. The compartment volumes were kept equal within the flow loops $[11,22]$. Twelve tangential compartments were used in each tank and the number of flow loops ranged from 8 to 15, which yielded in total from 10000 to 25000 compartments per tank. The circulation and exchange flow numbers, $N_{\mathrm{C}}$ and $N_{\mathrm{E}}$, were fitted to reproduce the local mixing times and tracer curves reported in literature.

The simulated macromixing time-scales were also compared to the mixing time-scales of equal-volume vessels having an aspect ratio of 1, a single impeller, a single feed point, and an equal power input. The correlation:

$$
\tau=\frac{5.3}{n} \frac{1}{N_{P}^{1 / 3}}\left(\frac{T}{D}\right)^{2}
$$

described in [23], was used to calculate these reference time-scales $\tau$ (T). In eq 4, $T$ is the vessel's diameter (L) and $N_{P}$ the turbulent power number assumed to have a value of 5.8 common to Rushton turbines [7]. The reference vessel's stirrer speed $n$ was adjusted to yield a similar power input as in the simulated tank by assuming that multi-impeller power equals single impeller power multiplied by the number of impellers, $N_{\mathrm{i}}$, while 
keeping the ratio of impeller and tank diameters the same. Under these assumptions, eq 4 led to the expression

$$
\tau=\frac{5.3}{n} \frac{(H / T)^{5 / 9}}{\left(N_{\mathrm{i}} N_{P}\right)^{1 / 3}}\left(\frac{T}{D}\right)^{2}
$$

which evaluates the reference time-scale $\tau$ as a function of the simulated tank's configuration. In eq 5, the definition of time-scale is not exactly the same as in the simulations (standard deviation $5 \%$ of mean), but the time-scales are nevertheless comparable.

\subsection{Software}

All calculations and simulations were performed with the Python 3.8.5 language (www.python.org) and the packages scipy 1.5.2 [24], numpy 1.19.2 [25], and pandas $1.1 .3[26,27]$.

\section{Results and discussion}

\subsection{Model fitting}

The proposed dispersion model (section 2.1) was found to reproduce previously published $[9,12,19,20]$ tracer curves by fitting its sole parameter, the dispersion coefficient $d$, to the experimentally determined local mixing times. Vessels equipped with one to four impellers and with differently positioned measurement probes were used to challenge the dispersion model. Table 1 summarizes the experiment configurations, and Figure 1 presents the experimental data and the model curves.

The model fitted the experimental data satisfyingly in the various geometries and configurations, and the tracer data were especially well reproduced in the bottom parts of the multi-impeller vessels. This comes as no surprise, as a cascade of ideal stirred tanks connected by exchange flows - a discretization of the dispersion model - can replicate experimental tracer curves [20] and has been used in multi-impeller mixing time correlations as well [28]. In the single-impeller configurations (panel D in Figure 1) the oscillations of the tracer signals could not be represented by the dispersion model, but the exponential approach towards a perfectly mixed state, the most important feature, was captured. These results suggest that the dispersion model describes mixing most accurately in tanks with multiple impellers.

\subsection{Placement of a single feed point}

Having confirmed that the simple dispersion model can describe macromixing in multi-impeller vessels, attention was shifted to its time-scales and geometrical features. Analogously to a classical residence time distribution based model of mixing [19], the 

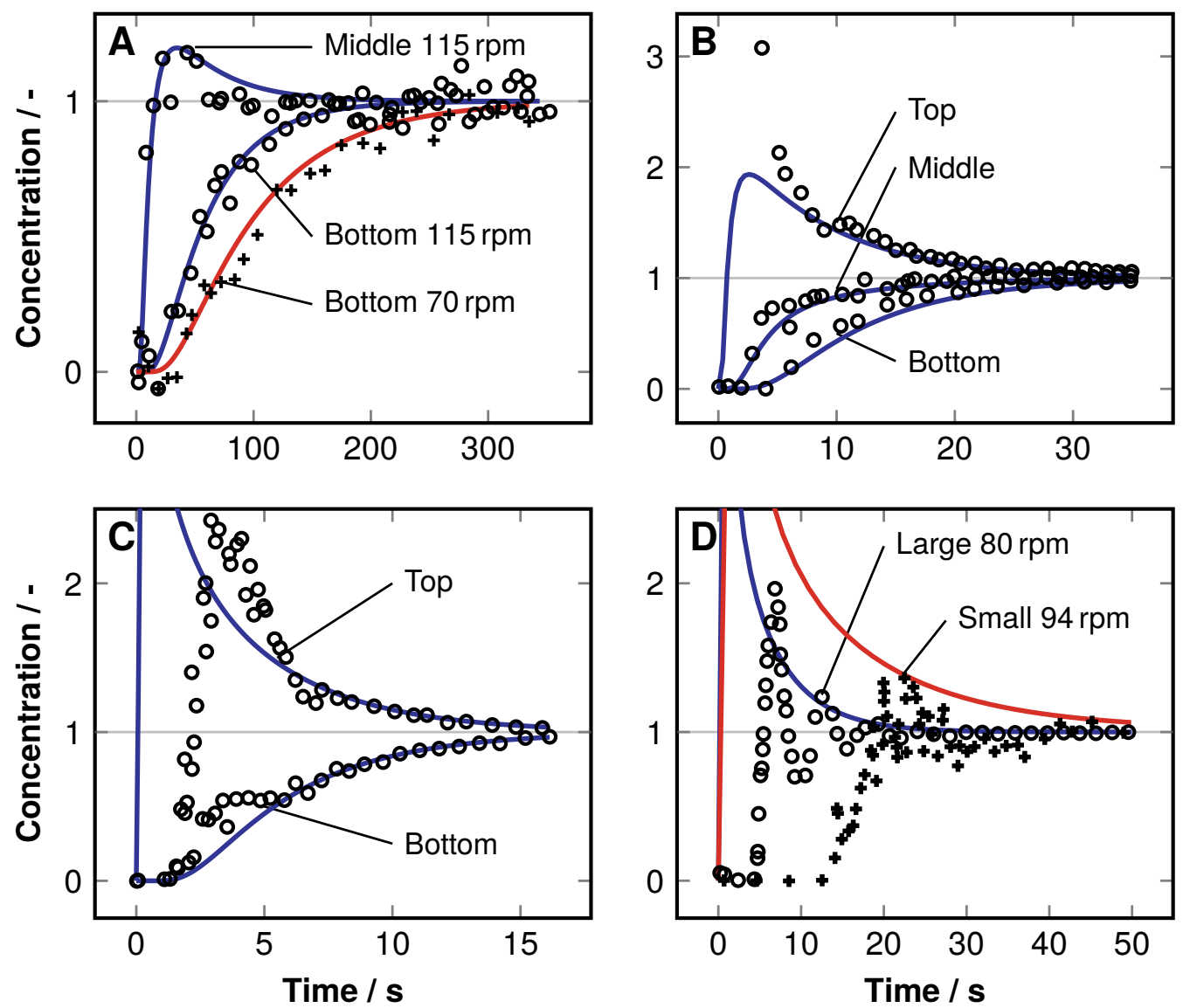

Figure 1: Cited experimental tracer curves and dispersion model fits. The tracer data were obtained from the published figures [9, 12, 19, 20] with WebPlotDigitizer [21]. The dispersion coefficients governing the model were fitted to the experimentally determined local mixing times. Solid curves represent the model fits, and experimental data points are marked with circles and crosses. Table 1 details the experimental setups. (A) $22 \mathrm{~m}^{3}$, four impellers [9]. Both $115 \mathrm{rpm}$ model curves have the same dispersivity. (B) $63 \mathrm{~L}$, three impellers [20]. All three signals were recorded simultaneously and fitted with a single dispersivity. (C) $17 \mathrm{~L}$, two impellers [12]. Both model curves were fitted with a single dispersivity. (D) Large vessel $1.4 \mathrm{~m}^{3}$, small vessel $140 \mathrm{~L}$. Both vessels had a single impeller [19]. 
Table 1: Cited tracer experiments and the fitted dispersivities. $V$ is the working volume, $n$ the stirrer speed, $t_{95}$ the local mixing time ( $95 \%$ homogeneity), $H / T$ the height-to-diameter ratio, $D / T$ the impeller-to-tank diameter ratio, $z_{0} / H$ the feed position relative to height, $z / H$ the measurement position relative to height, and $d$ the fitted dispersivity. The tracer data were obtained from the figures listed in the reference column.

\begin{tabular}{cccccccccc}
\hline $\begin{array}{c}\text { Fig 1 } \\
\text { panel }\end{array}$ & $\begin{array}{c}V \\
\mathrm{~m}^{3}\end{array}$ & $\begin{array}{c}n \\
\mathrm{rpm}\end{array}$ & $\begin{array}{c}t_{95} \\
\mathrm{~s}\end{array}$ & $\begin{array}{c}H / T \\
-\end{array}$ & $\begin{array}{c}D / T \\
-\end{array}$ & $\begin{array}{c}z_{0} / H \\
-\end{array}$ & $\begin{array}{c}z / H \\
-\end{array}$ & Reference & $\begin{array}{c}d \\
\mathrm{~cm}^{2} \mathrm{~s}^{-1}\end{array}$ \\
\hline $\mathrm{A}$ & 22 & 115 & 150 & 3 & 0.33 & 1 & $\begin{array}{c}0.15 \\
\text { [9] Fig 10 }\end{array}$ & 1000 \\
& & & & & & & 0.60 & [9] Fig 12b & \\
$\mathrm{A}$ & 22 & 70 & 250 & 3 & 0.33 & 1 & $\begin{array}{c}0.15 \\
\text { [9] Fig 10 }\end{array}$ & 610 \\
$\mathrm{~B}$ & 0.063 & 480 & 28 & 3 & 0.33 & 1 & 0.08 & [20] Fig 2 & 98 \\
& & & & & & & 0.42 & & \\
& & & & & & & 0.75 & & \\
$\mathrm{C}$ & 0.017 & 300 & 14 & 2 & 0.45 & 1 & 0.07 & {$[12]$ Fig 2 } & 49 \\
& & & & & & & 0.86 & & \\
$\mathrm{D}$ & 1.4 & 80 & 20 & 1 & 0.30 & 0.50 & 0.50 & [19] Fig 9c & 71 \\
$\mathrm{D}$ & 0.14 & 94 & 54 & 1 & 0.20 & 0.45 & 0.45 & {$[19]$ Fig 9b } & 5.9 \\
\hline
\end{tabular}

model (eq 3) contains first-order rate constants

$$
k^{2} \pi^{2} \frac{d}{H^{2}}, \quad k=1,2,3, \ldots
$$

of which the first $(k=1)$ limits the concentration's homogenization. These rate constants are associated with pre-exponential factors

$$
\cos \left(k \pi \frac{z_{0}}{H}\right) \cos \left(k \pi \frac{z}{H}\right), \quad k=1,2,3, \ldots
$$

which depend on the tracer injection and measurement positions, $z_{0}$ and $z$. It was then noticed that the $\cos \left(k \pi z_{0} / H\right)$ expressions in eq 7 would equal zero in all odd terms $(k=1,3,5, \ldots)$ if the feed point was brought to the middle $\left(z_{0} / H=0.5\right)$, and as a consequence, all odd terms of eq 3 would disappear and the limiting rate constant would be found in the second term $(k=2)$ associated with a 4-fold rate (eq 6). In other words, positioning the feed in the middle instead of the top or the bottom reduces the mixing time-scale to a quarter in theory.

Figure 2 shows how the feed point's position along the working height of the tank affects macromixing according to the dispersion model. As was anticipated in analyzing eqs 3 and 6, placing the feed point in the middle divides the mixing time-scale (standard deviation $5 \%$ of mean) by 4 . Similar results have been presented previously: injecting the tracer in the middle of a multi-impeller tank minimized mixing times in experiments [6] as well as in compartment model simulations [7]. The compartment model predictions were also partly verified with experiments [7]. Even though the observed mixing time reductions by a factor of approximately 2 were smaller than the model proposes, it is worth noting that mixing in real vessels stirred with multiple impellers behaved in accordance with the dispersion model's prediction. 


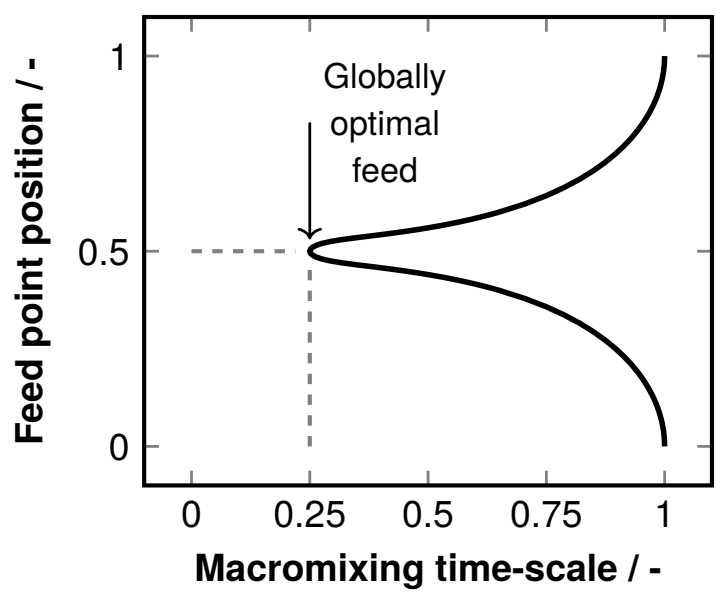

Figure 2: Optimal placement of a single feed point. The macromixing time-scale (concentration's standard deviation down to $5 \%$ of mean) is reduced to a quarter, if the feed point is placed axially in the middle instead of the top or the bottom.

\subsection{Effect of multiple feed points}

Motivated by the marked potential in reducing the macromixing time-scale by correct feed placement, the effect of using multiple such feed points was studied next. As the first-order rate constants (eq 6) of the dispersion model are inversely proportional to the vessel's working height squared, $H^{2}$, any reduction in height would greatly increase the mixing rate if the dispersion coefficient $d$ remained constant. Likewise, increasing the dispersivity would improve the rate in a given geometry. However, decreasing the height is unlikely to be a practical option and a greater dispersivity would require a higher power input for stirring. Could this square-relationship then be exploited without changing the vessel's geometry or the stirrer's power? The zero-gradient boundaries of the model (section 2.1) imply that dividing the working height into $N$ equally sized compartments with feed points in the centers will divide the axial domain length, the height $H$, by $N$ owing to symmetry. The limiting rate constant subsequently increases in proportion to the number of feed points squared, $N^{2}$ : for example two symmetrically placed feed points divide the mixing time-scale by 4 and three points by 9 . Compared with a single feed at the top, four ideally placed feed points divide the time-scale in theory by $64\left(4 \times 4^{2}\right)$. Thus, a closer inspection of these hypothetical order-of-magnitude mixing rate gains was warranted.

\subsection{Multi-port feed simulations}

The placement of multiple feed points was simulated with the compartment models (section 2.2) of the tanks described above (Table 1). As an initial design, the $N$ feed points were placed axially at positions $(1+2 N) /(2 N)$ relative to height as proposed in section 3.3 , radially at $2 / 3$ relative to radius, and tangentially evenly-spaced along the perimeter. As an example: with two feed points the dispersion model suggests axial 
locations $25 \%$ and $75 \%$ relative to height, with three points $17 \%, 50 \%$, and $83 \%$, and with four points $13 \%, 38 \%, 63 \%$, and $88 \%$. Radial positions were at first varied between $50 \%$ to $85 \%$, but the design was settled on $2 / 3 \approx 67 \%$, which performed well and lied conveniently between the tank and impeller perimeters. The tangential division did not have any notable effect in these simulations.

Figure 3 illustrates the theoretically optimal placement of one to four feed points and also presents simulation results in a $22 \mathrm{~m}^{3}$ bioreactor with four Rushton turbines [9] (A-115 rpm in Table 1), which confirm that considerable reductions in the macromixing time-scale are indeed attainable by dividing the feed equally into several points according to the dispersion model. With four symmetrically placed feed points the mixing rate was 29-fold relative to a single feed at the top, which falls short of the ideal 64-fold rate (section 3.3), but is nevertheless quite remarkable: the mixing time-scale (standard deviation $5 \%$ of mean) of a high aspect ratio $22 \mathrm{~m}^{3}$ reactor was brought from $140 \mathrm{~s}$ down to $4.9 \mathrm{~s}$ by replacing the single-point top feed by an ideal four-point feed. In comparison, the mixing time-scale in an equal-volume 1:1 aspect ratio vessel stirred with a single impeller and an equal power is expected (eq 5) to be on the order of $17 \mathrm{~s}$. Thus, using an appropriate multi-port feed can result in a mixing rate even greater than what is conventionally obtainable in a corresponding single-impeller configuration with a single feed point.

The simulated concentration fields in the $22 \mathrm{~m}^{3}$ reactor are illustrated with one, two, and four feed points in Figure 4. Using a conventional single-point top feed (left-most part of Figure 4) created a slowly-dissolving concentration gradient across the working height: the tracer concentration remained higher close to the feeding point and lower in the rest of the tank. Similar highly segregated concentration fields have been observed in many other simulations of tall multi-impeller vessels [3-5, 29], and the phenomenon has also been experimentally confirmed in bioreactors $[1,2,5]$. As can be seen in the middle and right-most parts of Figure 4, the segregation can be efficiently abolished by using as little as two appropriately placed feed points, leading to superior homogenization. The difference between the macromixing performance of a single top feed and of multiple optimally positioned ones can be understood in terms of mass transfer, which is driven by concentration gradients across interfacial areas. Even though a single feed at the top resulted in a gradient throughout the vessel, the apparent interfacial area was limited to the tank's cross-section, whereas multiple appropriately placed feed points established several apparent interfaces for turbulent transfer.

Even over 30-fold macromixing rates were seen in simulations of the $22 \mathrm{~m}^{3}$ reactor by using more than four feeds, but the additional rate enhancements were not substantial beyond four points. The maximal number of feed points was then settled at four for the following simulations, as using more could complicate practical implementation of equipment with only nominally improved rates. Placing the feeds at the same heights as the impellers was also simulated, but this feed placement led to synchronization of the impeller-wise circulation flows and lowered the mixing rates. Experience and previous research [16] has shown that asynchronous circulation is preferable to synchronous.

Multi-port feed simulations were carried out also in the other vessels (Table 1), and 


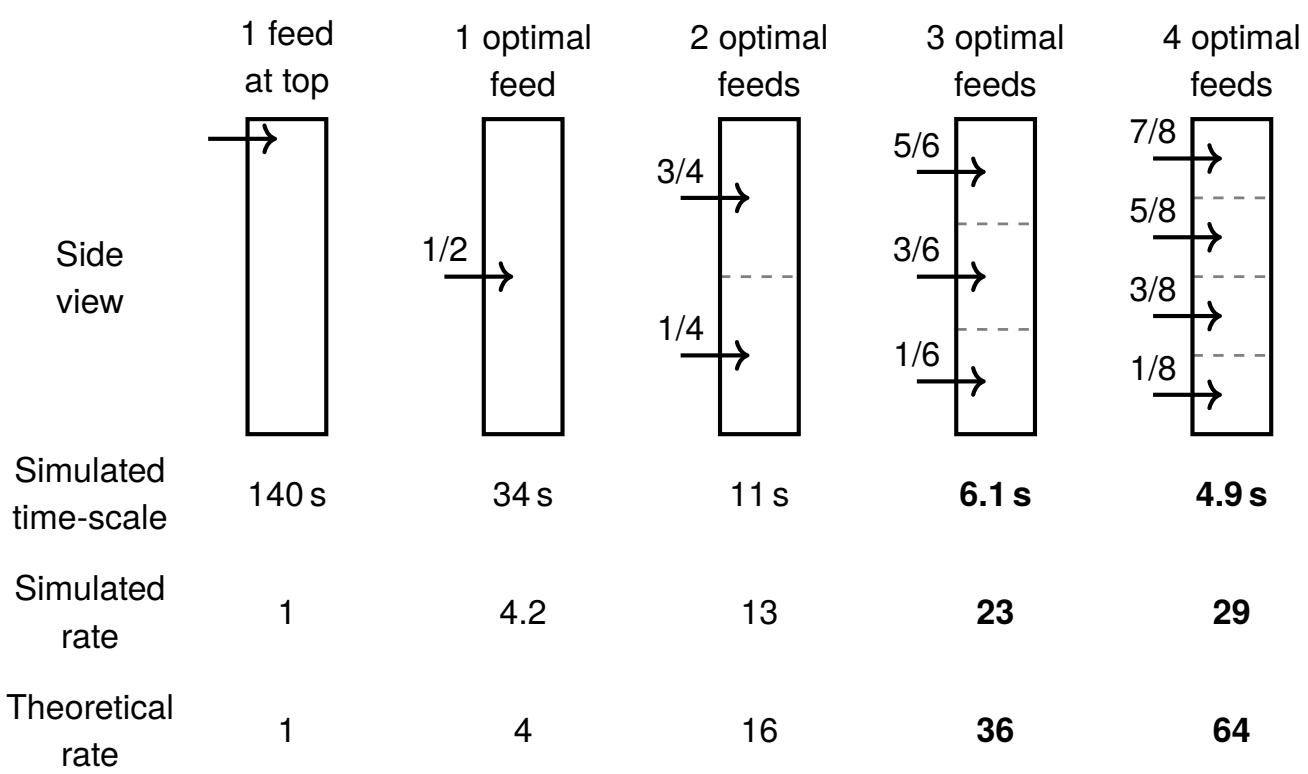

Figure 3: Optimally placed multiple feed points. The side view illustrates how the axial domain is divided (dashed lines) into equal subdomains with a centrally placed feed point in each. The arrows represent the feed positions relative to the liquid height. The macromixing time-scales were simulated with a compartment model of a $22 \mathrm{~m}^{3}$ working volume with an aspect ratio of 3 (A-115 rpm in Table 1). The time-scale refers to the time required to reduce the concentration's standard deviation down to $5 \%$ relative to mean. Simulated rate is the factor by which the time-scale has been divided compared with a single feed point at the top. Corresponding theoretical rates (section 3.3) predicted by the dispersion model are shown for reference.

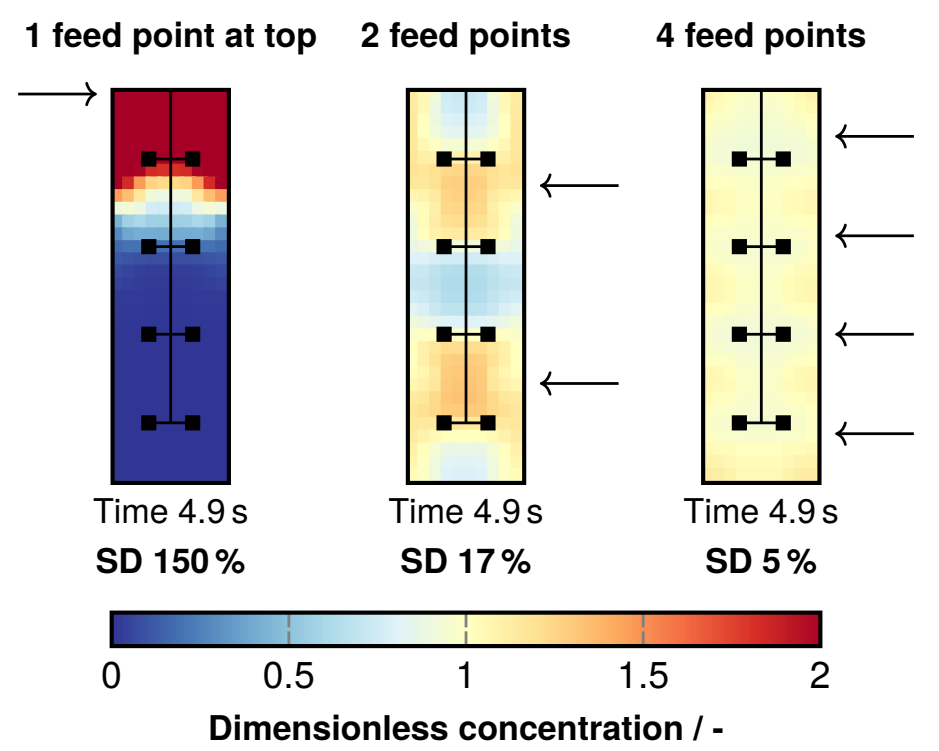

Figure 4: Simulated concentration fields in a $22 \mathrm{~m}^{3}$ bioreactor with four impellers (A-115 rpm in Table 1) $4.9 \mathrm{~s}$ after pulse addition of tracer. SD stands for standard deviation relative to mean. 
Table 2: Simulated macromixing time-scales (s). The time-scale is the time required to reduce the concentration's standard deviation to $5 \%$ of mean. The number of feed points is denoted by $N$, and the letters, volumes, and stirrer speeds identify the vessels (Table 1 and Figure 1). The bottom row (1:1) shows reference mixing time-scales calculated with eq 5 in an equal-volume tank with 1:1 aspect ratio, one impeller, and an equal power input.

\begin{tabular}{ccccccc}
\hline$N$ & $\mathrm{~A}$ & $\mathrm{~A}$ & $\mathrm{~B}$ & $\mathrm{C}$ & $\mathrm{D}$ & $\mathrm{D}$ \\
& $22 \mathrm{~m}^{3}$ & $22 \mathrm{~m}^{3}$ & $63 \mathrm{~L}$ & $17 \mathrm{~L}$ & $1.4 \mathrm{~m}^{3}$ & $0.14 \mathrm{~m}^{3}$ \\
& $115 \mathrm{rpm}$ & $70 \mathrm{rpm}$ & $480 \mathrm{rpm}$ & $300 \mathrm{rpm}$ & $80 \mathrm{rpm}$ & $94 \mathrm{rpm}$ \\
\hline 1 (top) & 143 & 238 & 30.2 & 13.6 & 25.0 & 52.2 \\
1 & 34.0 & 56.9 & 7.17 & 5.14 & 17.1 & 35.3 \\
2 & 10.6 & 17.9 & 2.05 & 2.43 & 8.99 & 24.5 \\
3 & 6.13 & 10.1 & 1.92 & 2.45 & 12.8 & 26.6 \\
4 & 4.91 & 8.10 & 1.93 & 3.88 & 8.14 & 25.8 \\
$1(1: 1)$ & 16.5 & 27.2 & 4.24 & 3.33 & 24.6 & 45.3 \\
\hline
\end{tabular}

the simulation results are summarized in Table 2. Compared with using a single top feed, at highest a 16-fold macromixing rate was obtained in the three-impeller configuration (B) and a 5.6-fold rate with two impellers (C). The single-impeller vessels (D) experienced approximately doubled mixing rates. As described in section 3.1, the dispersion model's capability of reproducing experimental tracer curves seemed to be proportional to the number of impellers (Figure 1). Here a similar observation was made: in simulations the relative benefit of using multiple feed points correlated with the number of impellers. It is therefore tempting to hypothesize that using several impellers makes a vessel's mixing behaviour approach the ideal represented by the simple dispersion model. Even though the improvements in the single-impeller tanks were modest in comparison to multi-impeller configurations, the mixing rates were nevertheless doubled. It would be interesting to study in the future, whether optimizing [8] the feed point placement in single-impeller configurations would yield further gains. In previously published experiments [8], the use of two feed points resulted in 2.9 to 6.6-fold mixing rates in pneumatically agitated reactors. Interestingly, the corresponding two-feed-point rates were here as high as 5.6 to 15 -fold in the simulated multi-impeller vessels (A, B, and C).

\subsection{Implications}

The presented simulation results imply that the feed-related gradients found for example in large-scale bioprocesses [1-5] could be diminished considerably by dividing the feed into multiple symmetrically positioned points. Similar benefits could also be anticipated in other contexts. An order-of-magnitude reduction in the macromixing time-scale could make large-scale equipment behaviour resemble laboratory-scale performance, which in turn should alleviate mixing-associated problems $[5,30]$ encountered in the scale-up. However, in a bioprocessing context the prevention of contamination would also need to be accounted for upon implementation. 
If the micromixing time-scales need to prioritized, the feed points could be brought closer to the impellers [31]. Considering that the turbulence dissipation rates are highest at the impeller discharges [32], it could also be expected that a multi-impeller vessel should have a more homogeneous distribution of turbulence dissipation and thus more homogeneous mass transfer characteristics. Hence, a multi-impeller tank with an optimal multi-point feed might be comparable or even superior to a single-impeller vessel in both micro- and macromixing terms, but experimental verification is needed.

Even though using multiple feed points is inherently more complex than using a single one, implementing such a device should not be technically overwhelming. The most challenging aspects are likely to be found in ensuring equal flow rates in the multiple ports and in preventing contamination where it is of concern. An ideally positioned multi-point feed has great prospects in removing macromixing limitations, and it is hoped that this potential could be realized experimentally and ultimately in production.

\section{Conclusions}

Based on the presented results, the following conclusions are drawn to answer the research questions:

1. Macromixing in multi-impeller vessels can be described by a transient diffusion equation, the dispersion model, in the absence of complete flow field information.

2. A single feed point should be placed in the middle of the vessel's working height.

3. Multi-port feeding is optimal for macromixing in high aspect ratio tanks stirred with multiple impellers.

4. The multiple feed points should be placed in the middles of equal-sized axial subdivisions. The radial position can initially be set halfway between the impeller and tank perimeters.

\section{Author contributions}

PL: Conceptualization, Investigation, Methodology, Software, Writing - original draft, Writing - reviewing and editing. JK: Supervision, Writing - reviewing and editing. VS: Supervision, Writing - reviewing and editing. All authors read and approved the final manuscript.

\section{Funding}

This work was supported by Tampere University of Technology Graduate School and Academy of Finland (grant no. 310188). 


\section{Declaration of interests}

The authors declare that they have no known competing financial interests or personal relationships that could have appeared to influence the work reported in this paper.

\section{References}

[1] Bylund, F.; Collet, E.; Enfors, S.; Larsson, G. Substrate gradient formation in the large-scale bioreactor lowers cell yield and increases by-product formation. Bioprocess Engineering 1998, 18, 171-180, DOI: 10.1007/s004490050427.

[2] Xu, B.; Jahic, M.; Blomsten, G.; Enfors, S. Glucose overflow metabolism and mixedacid fermentation in aerobic large-scale fed-batch processes with Escherichia coli. Applied Microbiology and Biotechnology 1999, 51, 564-571, DOI: 10.1007 / s002530051433.

[3] Vlaev, D.; Mann, R.; Lossev, V.; Vlaev, S. D.; Zahradnik, J.; Seichter, P. MacroMixing and Streptomyces fradiae: Modelling Oxygen and Nutrient Segregation in an Industrial Bioreactor. Chemical Engineering Research and Design 2000, 78, 354-362, DOI: 10.1205/026387600527473.

[4] Pigou, M.; Morchain, J. Investigating the interactions between physical and biological heterogeneities in bioreactors using compartment, population balance and metabolic models. Chemical Engineering Science 2015, 126, 267-282, DOI: $10.1016 / \mathrm{j}$. ces . 2014.11 .035 .

[5] Nadal-Rey, G.; McClure, D. D.; Kavanagh, J. M.; Cornelissen, S.; Fletcher, D. F.; Gernaey, K. V. Understanding gradients in industrial bioreactors. Biotechnology Advances 2021, 107660, DOI: 10.1016/j . biotechadv . 2020. 107660.

[6] Cronin, D. G.; Nienow, A. W.; Moody, G. W. An Experimental Study of Mixing in a Proto-Fermenter Agitated by Dual Rushton Turbines. Food and Bioproducts Processing 1994, 72, 35-40.

[7] Vrábel, P.; van der Lans, R. G. J. M.; Cui, Y. Q.; Luyben, K. C. A. M. Compartment Model Approach: Mixing in Large Scale Aerated Reactors with Multiple Impellers. Chemical Engineering Research and Design 1999, 77, 291-302, DOI: 10. 1205/ Q26387699526223.

[8] Fu, C.; Lu, S.; Wu, W. Optimal feeding for tower-type reactors. AIChE Journal 2005, 51, 713-724, DOI: 10.1002/aic. 10346.

[9] Cui, Y. Q.; van der Lans, R. G. J. M.; Noorman, H. J.; Luyben, K. C. A. M. Compartment Mixing Model for Stirred Reactors with Multiple Impellers. Chemical Engineering Research and Design 1996, 74, 261-271. 
[10] Vrábel, P.; van der Lans, R. G. J. M.; Luyben, K. C. A. M.; Boon, L.; Nienow, A. W. Mixing in large-scale vessels stirred with multiple radial or radial and axial up-pumping impellers: modelling and measurements. Chemical Engineering Science 2000, 55, 5881-5896, DOI: 10.1016/S0009-2509(00)00175-5.

[11] Zahradník, J.; Mann, R.; Fialová, M.; Vlaev, D.; Vlaev, S. D.; Lossev, V.; Seichter, P. A networks-of-zones analysis of mixing and mass transfer in three industrial bioreactors. Chemical Engineering Science 2001, 56, 485-492, DOI: 10.1016/ S0009-2509(00)00252-9.

[12] Delafosse, A.; Collignon, M.; Calvo, S.; Delvigne, F.; Crine, M.; Thonart, P.; Toye, D. CFD-based compartment model for description of mixing in bioreactors. Chemical Engineering Science 2014, 106, 76-85, DOI: 10.1016/j . ces . 2013. 11.033.

[13] Delafosse, A.; Calvo, S.; Collignon, M.; Delvigne, F.; Crine, M.; Toye, D. EulerLagrange approach to model heterogeneities in stirred tank bioreactors - Comparison to experimental flow characterization and particle tracking. Chemical Engineering Science 2015, 134, 457-466, DOI: 10.1016/j . ces .2015 . 05 . 045.

[14] Holmes, D. B.; Voncken, R. M.; Dekker, J. A. Fluid flow in turbine-stirred, baffled tanks-I: Circulation time. Chemical Engineering Science 1964, 19, 201-208, DOI: $10.1016 / 0009-2509$ (64)85030-2.

[15] Voncken, R. M.; Holmes, D. B.; den Hartog, H. W. Fluid flow in turbine-stirred, baffled tanks-II: Dispersion during circulation. Chemical Engineering Science 1964, 19, 209-213, DOI: 10.1016/0009-2509(64)85031-4.

[16] Giona, M.; Paglianti, A.; Cerbelli, S.; Pintus, S.; Adrover, A. Tracer Dispersion in Stirred Tank Reactors: Asymptotic Properties and Mixing Characterization. The Canadian Journal of Chemical Engineering 2002, 80, 580-590, DOI: 10.1002/ cjce. 5450800421.

[17] Mayr, B.; Horvat, P.; Moser, A. Engineering approach to mixing quantification in bioreactors. Bioprocess Engineering 1992, 8, 137-143, DOI: 10 . 1007 / BFO1254229.

[18] Cole, K. D.; Beck, J. V.; Haji-Sheikh, A.; Litkouhi, B., Heat Conduction Using Green's Functions, 2nd ed.; CRC Press: 2010, DOI: 10.1201/9781439895214.

[19] Khang, S. J.; Levenspiel, O. New scale-up and design method for stirrer agitated batch mixing vessels. Chemical Engineering Science 1976, 31, 569-577, DOI: 10. 1016/0009-2509(76)80020-6.

[20] Kasat, G. R.; Pandit, A. B. Mixing Time Studies in Multiple Impeller Agitated Reactors. The Canadian Journal of Chemical Engineering 2004, 82, 892-904, DOI: $10.1002 /$ cjce. 5450820504 .

[21] Rohatgi, A. Webplotdigitizer: Version 4.4, 2020, https : //automeris . io/ WebPlotDigitizer. 
[22] Hristov, H.; Mann, R.; Lossev, V.; Vlaev, S. D.; Seichter, P. A 3-D Analysis of Gas-Liquid Mixing, Mass Transfer and Bioreaction in a Stirred BioReactor. Food and Bioproducts Processing 2001, 79, 232-241, DOI: 10. 1205/ Q96030801753252306.

[23] Nienow, A. W. On impeller circulation and mixing effectiveness in the turbulent flow regime. Chemical Engineering Science 1997, 52, 2557-2565, DOI: 10. 1016/ S0009-2509 (97)00072-9.

[24] Virtanen, P. et al. SciPy 1.0: fundamental algorithms for scientific computing in Python. Nature Methods 2020, 17, 261-272, DOI: 10 . 1038/s41592-019-06862.

[25] Harris, C. R. et al. Array programming with NumPy. Nature 2020, 585, 357-362, DOI: $10.1038 / \mathrm{s} 41586-020-2649-2$.

[26] The pandas development team Pandas, version 1.1.3, 2020, DOI: 10 . 5281/ zenodo. 4067057.

[27] McKinney, W. In Proceedings of the 9th Python in Science Conference, 2010, pp 51-56, DOI: 10.25080/Majora-92bf1922-00a.

[28] Magelli, F.; Montante, G.; Pinelli, D.; Paglianti, A. Mixing time in high aspect ratio vessels stirred with multiple impellers. Chemical Engineering Science 2013, 101, 712-720, DOI: $10.1016 / \mathrm{j}$. ces .2013.07.022.

[29] Montante, G.; Magelli, F. Liquid Homogenization Characteristics in Vessels Stirred with Multiple Rushton Turbines Mounted at Different Spacings: CFD Study and Comparison with Experimental Data. Chemical Engineering Research and Design 2004, 82, 1179-1187, DOI: 10.1205/cerd.82 . 9.1179.44163.

[30] Enfors, S. et al. Physiological responses to mixing in large scale bioreactors. Journal of Biotechnology 2001, 85, 175-185, DOI: 10. 1016/SQ168-1656(Q0) 00365-5.

[31] Patterson, G. K.; Paul, E. L.; Kresta, S. M.; Etchells, A. W. In Handbook of Industrial Mixing: Science and Practice, Paul, E. L., Atiemo-Obeng, V. A., Kresta, S. M., Eds.; John Wiley \& Sons, Ltd.: 2003; Chapter 13, pp 755-867, DOI: 10.1002/0471451452. ch13.

[32] Baldi, S.; Yianneskis, M. On the quantification of energy dissipation in the impeller stream of a stirred vessel from fluctuating velocity gradient measurements. Chemical Engineering Science 2004, 59, 2659-2671, DOI: 10.1016/j . ces . 2004.03 .021 . 